\title{
Expression of phosphoproteins and amelotin in teeth
}

\author{
BEAT TRUEB ${ }^{1}$, SARA TAESCHLER ${ }^{1}$, CHRISTOF SCHILD $^{1}$ and NIKLAUS P. LANG ${ }^{2}$ \\ ${ }^{1}$ ITI Research Institute for Dental and Skeletal Biology, ${ }^{2}$ Department of Periodontology and \\ Fixed Prosthodontics, University of Bern, CH-3010 Bern, Switzerland
}

Received July 10, 2006; Accepted September 8, 2006

\begin{abstract}
The organic material of our teeth consists of collagens and a number of calcium-binding phosphoproteins. Six of these phosphoproteins have recently been grouped in the family of the SIBLINGs (small integrin-binding ligand, $\mathrm{N}$-linked glycoproteins), namely osteopontin, bone sialoprotein, dentin matrix protein (DMP1), dentin sialophosphoprotein (DSPP), matrix extracellular phosphoglycoprotein (MEPE) and enamelin. We prepared a cDNA library from rat incisors in order to identify the genes involved in tooth formation. The library was screened by subtractive hybridization with two probes; one specific for teeth, the other for bone. We found that the vast majority of the clones from our library were expressed at similar levels in bone and teeth, demonstrating the close relationship of the two tissues. Only $7 \%$ of all the clones were expressed in a tooth-specific fashion. These included clones for the enamel proteins; amelotin, amelogenin, ameloblastin and enamelin; for the dentin proteins DSPP and DMP1; and for the intermediate filament protein cytokeratin 13 . Several typical bone proteins, including collagen I, osteocalcin, alkaline phosphatase and FATSO, were also expressed at significantly higher levels in teeth than in bone, probably due to the extreme growth rate of rat incisors. The amino acid sequence of rat amelotin showed $62 \%$ identity with the sequence from humans. It was expressed considerably later than the other enamel proteins, suggesting that amelotin may serve a function different from those of amelogenin, ameloblastin and enamelin.
\end{abstract}

\section{Introduction}

Recent studies suggest that biomimetic peptides can be used in the design of prosthetic implants to improve osseointegration of the synthetic material in the jaw. Such peptides can be derived from extracellular matrix proteins that are known to induce cell adhesion. Often these proteins harbor the tripeptide

Correspondence to: Dr Beat Trueb, ITI Research Institute, University of Bern, Murtenstrasse 35, P.O. Box 54, CH-3010 Bern, Switzerland

E-mail: beat.trueb@iti.unibe.ch

Key words: amelotin, dentin, enamel, phosphoproteins, rat incisors, SIBLING, tooth formation sequence RGD, which interacts with integrin receptors of cell surfaces.

Teeth consist of dentin, cementum and enamel. Dentin and cementum closely resemble bone in their molecular composition $(1,2)$. These tissues consist of mineral crystals that are embedded in a rich extracellular matrix. The predominant protein of the extracellular matrix is collagen type I, contributing up to $90 \%$ of the organic material of mineralized dentin. The rest is made up by various glycoproteins. The glycoproteins that are found in dentin as well as in bone include osteonectin, osteocalcin, matrix Gla protein, osteopontin and bone sialoprotein (IBSP). The glycoproteins that are preferentially expressed in dentin are dentin sialophosphoprotein (DSPP) and dentin matrix protein (DMP1). The enamel of the crown differs from dentin and cementum in that it contains no collagens. The principal components of this tissue are amelogenin (AMELX), ameloblastin (AMBN) and enamelin (ENAM) (3).

Fisher and colleagues (4) have suggested that the calcium-binding phosphoproteins of teeth and bone should be grouped in a separate family, termed the SIBLING proteins (small integrin-binding ligand, N-linked glycoproteins). Today, this family comprises six proteins: bone sialoprotein, DMP1, DSPP, enamelin, matrix extracellular phosphoglycoprotein (MEPE) and osteopontin (5). A direct comparison of their amino acid sequences does not reveal much similarity. However, a detailed analysis of the corresponding genes shows a closer relationship. All genes are clustered on the long arm of human chromosome 4 . They start with a noncoding exon, followed by the exon for the signal peptide. The majority of the protein is encoded by the last one or two exons and contains the integrin-binding sequence RGD. When the sequence of the human genome was completed, it became apparent that the ameloblastin gene is also located in the SIBLING cluster next to the enamelin gene. It was therefore concluded that the vertebrate genes for all secreted, calcium-binding phosphoproteins may have evolved from a common ancestor by tandem gene duplication $(6,7)$.

We have set out to analyze the molecular composition of teeth. Since most of these molecules are insoluble in physiological buffers, we analyzed the corresponding mRNAs and prepared a tooth-specific cDNA library. As a model system, we chose the rat incisor. Rat incisors erupt at day 810 after birth and grow throughout life at a rate of $2.8 \mathrm{~mm}$ per week (8). This makes the rat incisor a popular model to be analyzed by molecular biological techniques. 


\section{Materials and methods}

RNA isolation and Northern blotting. Teeth were extracted from 1- to 56-day-old Wistar rats and directly homogenized in guanidinium buffer utilizing a polytron (Kinematica AG, Lucerne, Switzerland) or a freezer mill (Spex CertiPrep, Metuchen, NJ). For some experiments, the pulpa was removed from the teeth and treated separately as a source enriched in odontoblast RNA. RNA from ameloblasts was obtained by soaking the incisors in Eppendorf tubes filled halfway with guanidinium buffer. For this purpose, the teeth were put into the tubes with their tips down, and special care was taken that the most apical portions were not covered with buffer so that no buffer reached the root canal. All samples were extracted with one volume of phenol chloroform before the RNA was isolated by the guanidinium isothiocyanate method (9) utilizing the RNeasy kit from Qiagen GmbH (Hilden, Germany). The purified RNA was separated on $1 \%$ agarose gels in the presence of formaldehyde and then transferred to nylon membranes by vacuum blotting (10). The blots were hybridized at $42^{\circ} \mathrm{C}$ with radioactively labeled cDNA probes in a buffer containing $50 \%$ formamide. These probes had been labeled by the random primed oligolabeling method (11). The blots were washed at regular stringency (1X SSC) and exposed to BioMax MS X-ray film (Kodak, Rochester, NY) or analyzed with a phosphorimager (Storm 840, Molecular Dynamics, Sunnyvale, CA). For some applications, poly(A) RNA was prepared from total RNA by chromatography on oligo d(T) beads (Oligotex, Qiagen $\mathrm{GmbH}$ ).

Preparation of cDNA library. The SuperScript system from Invitrogen Life Technologies was used to construct a toothspecific cDNA library. An oligo dT primer containing a Not I restriction site was annealed to poly(A) RNA from the incisors of 4-week-old Wistar rats and first-strand synthesis was performed with SuperScript II reverse transcriptase. The second strand was constructed with DNA polymerase I using Gubler-Hoffman cloning (10). Double-stranded cDNA was provided with Sal I adapters and fractionated by gel filtration. The size selected cDNA was cloned into the Sal INot I restriction site of the vector pSPORT1 (Invitrogen). The final plasmids were transfected into E. coli $\mathrm{DH} 10 \mathrm{~B}$ and plated onto ampicillin containing agar plates.

Library screening and gene arrays. To search for toothspecific clones, the bacterial colonies were spotted in a $8 \times 12$ format onto nylon filters. Two identical sets of filters were hybridized in parallel with two radioactive cDNA probes. One was prepared from poly(A) RNA of rat teeth (incisors from 4-week-old rats), the other from poly(A) RNA of rat bone (femur of a 4-week-old rat). The probes were labeled by annealing of an oligo(dT) primer to the RNA, followed by reverse transcription at $50^{\circ} \mathrm{C}$ in the presence of $\left[\alpha^{-32} \mathrm{P}\right] \mathrm{dCTP}$. The resulting cDNAs were purified by size chromatography as described (12). After hybridization, the filters were washed and analyzed with a phosphorimager. Images obtained with a set of identical filters were superimposed with Adobe Photoshop 7.0. Dots observed with the toothspecific probe were loaded into the red channel, dots

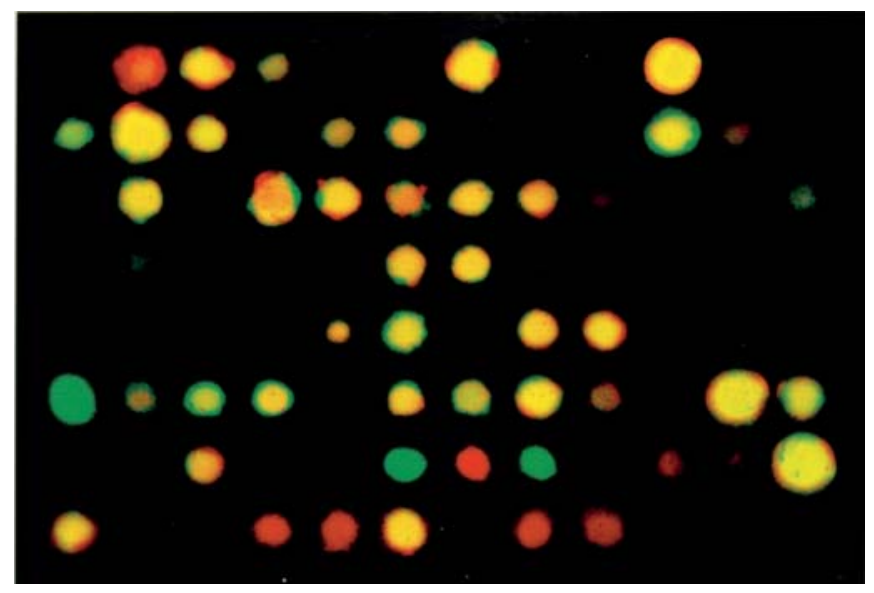

Figure 1. Gene array containing clones from the tooth-specific cDNA library. The clones were spotted by hand onto nylon membranes and hybridized with two probes specifically prepared from rat teeth and rat bone, respectively. Red, clones reacting with the probe from teeth; green, clones reacting with the probe from bone; yellow dots, clones that reacted equally with either probe. For further details see 'Materials and methods'.

observed with the bone-specific probe were loaded into the green channel. Clones of interest were sequenced by the dideoxy chain termination method using a cycle sequencer from Applied Biosystems (Foster City, CA). DNA sequences were analyzed with the GCG computer program from Accelrys Software Inc. (Cambridge, UK).

\section{Results}

Tooth-specific cDNA library. The cDNA library prepared from the rat incisors showed a complexity of $2 \times 10^{6}$ independent cDNA clones. Many of these clones coded for typical proteins of teeth and bones, such as amelogenin, collagen I, osteocalcin and osteopontin. However, our library also contained a great number of clones for ubiquitous proteins, such as ribosomal protein L10, B-actin and filamin-B. To identify tooth-specific clones, we prepared gene arrays. The clones of our library were spotted onto nylon membranes. Two identical sets of membranes were hybridized in parallel with two specific cDNA probes. One probe was prepared from teeth, the other from bone. The hybridization signals were analyzed with a computer, whereby signals obtained with the tooth-specific probe were plotted in red and signals obtained with the bone-specific probe were plotted in green (Fig. 1). When the two images were superimposed it became evident that nearly half of all the clones produced no signal, suggesting that they were expressed at extremely low levels. The vast majority of the other clones were plotted in yellow, indicating that they were expressed at similar levels in teeth and in bone. About $7 \%$ of the clones were plotted in red, suggesting that they were expressed specifically in teeth (Fig. 1). The sequencing of these clones demonstrated that they coded primarily for amelogenin, ameloblastin, DSPP, collagen I and osteocalcin. Single clones were found for cytokeratin 13, alkaline phosphatase, the rat homologue of mouse amelotin (AMTN, accession no. AM231717) and rat FATSO (accession no. AM233906). 


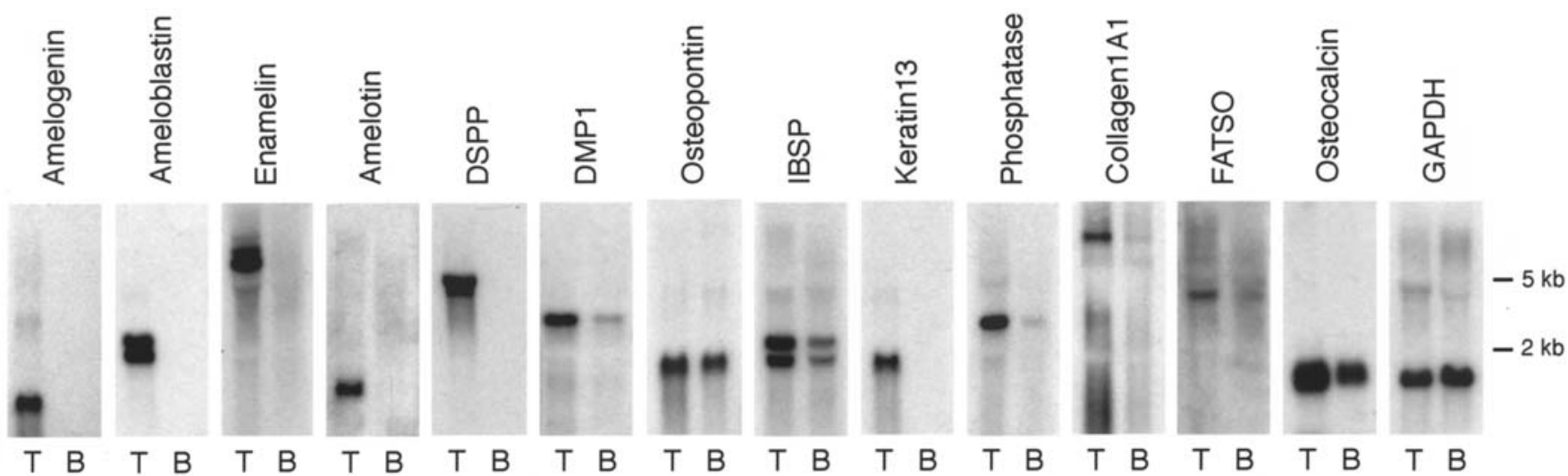

Figure 2. Northern blots with selected cDNA clones. Total RNA from teeth (T) and bone (B) was separated on agarose gels, transferred to nylon membranes and hybridized with radioactively labeled clones from the cDNA library. A blot hybridized with a probe for glyceraldehyde dehydrogenase (GAPDH) is included as a control for equal loading. The migration positions of the ribosomal RNAs are indicated in the right margin.

Specificity of clones. The specificity of our clones was demonstrated on Northern blots with RNA from incisors and RNA from femur (Fig. 2). The signals obtained with our probes for collagen I, alkaline phosphatase, FATSO and osteocalcin were found to be much stronger in the toothspecific lane than in the bone-specific lane. This can be explained by the fact that these genes are transcribed at much higher rates in incisors than in bone. The signals for amelogenin, ameloblastin, amelotin, DSPP and cytokeratin 13 were specifically found in the lane with RNA from teeth.

Ameloblastin, DSPP and osteopontin belong to the family of the secreted, Ca-binding phosphoproteins. We therefore became interested in the expression of the remaining members of this family, namely enamelin, bone sialoprotein, DMP1 and MEPE. Screening of our library with short EST clones for these proteins yielded full-length or nearly full-length clones for rat DMP1, bone sialoprotein and enamelin. In contrast, we obtained no clones for MEPE. Northern blots with the additional probes demonstrated that enamelin was expressed exclusively in teeth, whereas DMP1 and bone sialoprotein were expressed in teeth and bone (Fig. 2).

To discriminate whether the tooth-specific proteins were produced by the ameloblasts or by the odontoblasts, we prepared Northern blots with cell specific RNA (Fig. 3). The genes for amelogenin, ameloblastin and enamelin were found to be expressed primarily by the ameloblasts located at the surface of the teeth. However, small amounts of the transcripts could also be identified in the RNA preparation from the odontoblasts. Only amelotin appeared to be expressed exclusively by the ameloblasts. In contrast, transcripts for DSPP and DMP1 were found in the RNA preparation from the odontoblasts but not in that from the ameloblasts (Fig. 3).

Sequence of rat amelotin. Since this is the first report about amelotin from rats, we compared its amino acid sequence with the sequences from other species (Fig. 4). Amelotin from dogs, whose sequence can be predicted from the completed genomic sequence, was included in this alignment. Rat amelotin showed $62 \%$ sequence identity with human amelotin and $89 \%$ identity with mouse amelotin (Fig. 4). The central part of the protein exhibited a slightly higher

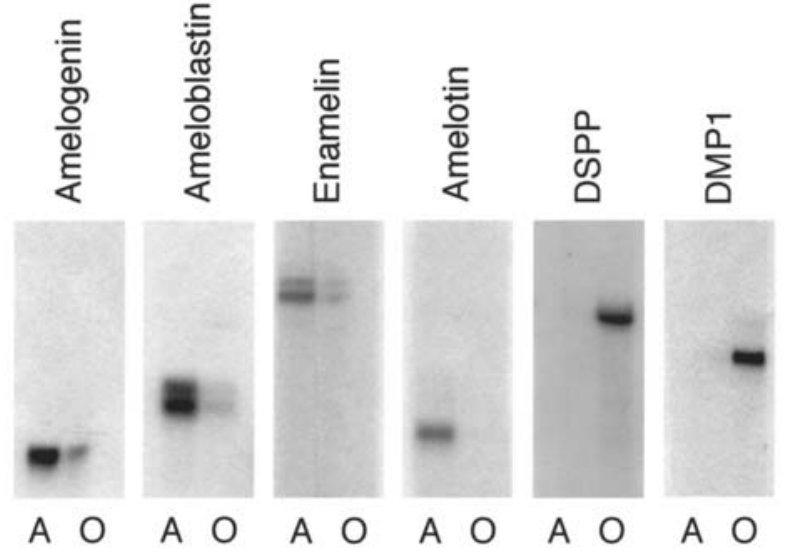

Figure 3. Differential expression of the tooth-specific genes by ameloblasts and by odontoblasts. Samples enriched with RNA from ameloblasts (A) were obtained from the surface of rat incisors; samples enriched with RNA from odontoblasts $(\mathrm{O})$ from the pulpa of the teeth. The samples were processed for Northern blotting as described in the legend of Fig. 2.

conservation than the $\mathrm{N}$-terminal or the $\mathrm{C}$-terminal part but there was no obvious, well-conserved domain, which would allow speculation about a putative function of the novel protein.

Expression in the mandible. The expression of all the toothspecific proteins was determined during the development of an entire jaw. For this experiment, RNA was extracted from the mandibles of newborn rats between day 1 and week 8 and analyzed by Northern blotting (Fig. 5A). This experiment showed clearly distinct expression curves for the six toothspecific genes. Expression of the enamel proteins amelogenin, ameloblastin and enamelin was already detectable at birth, then increased and formed a peak one week after birth before it declined again. Expression of DSPP and amelotin was barely visible at birth, but then increased and reached a maximum two weeks after birth. DMP1 exhibited an expression curve intermediate between that of the enamel proteins and that of DSPP. Its expression was quite high at birth, then increased further and formed a peak two weeks after birth (Fig. 5B). 


\begin{tabular}{|c|c|c|}
\hline $\begin{array}{l}\text { Mouse } \\
\text { Rat } \\
\text { Dog } \\
\text { Human }\end{array}$ & $\begin{array}{l}1 \\
1\end{array}$ & 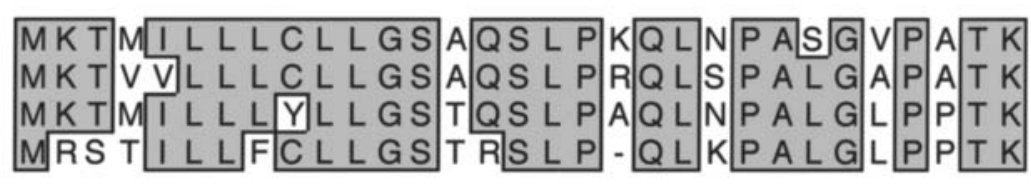 \\
\hline bus & $\begin{array}{l}32 \\
32 \\
32 \\
31\end{array}$ & 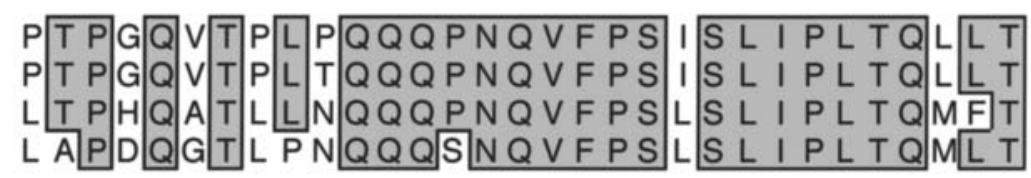 \\
\hline $\begin{array}{l}\mathrm{t} \\
\mathrm{aman}\end{array}$ & $\begin{array}{l}63 \\
63 \\
63 \\
62\end{array}$ & 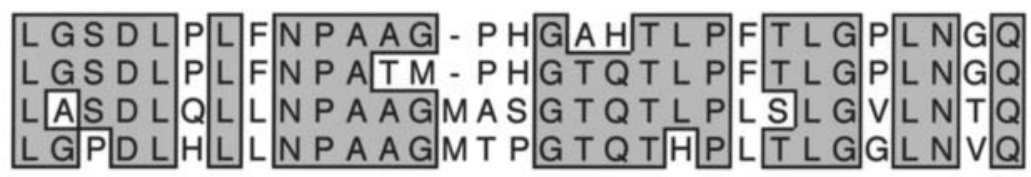 \\
\hline $\begin{array}{l}\text { ouse } \\
\text { at } \\
\text { og } \\
\text { uman }\end{array}$ & $\begin{array}{l}93 \\
93 \\
94 \\
93\end{array}$ & 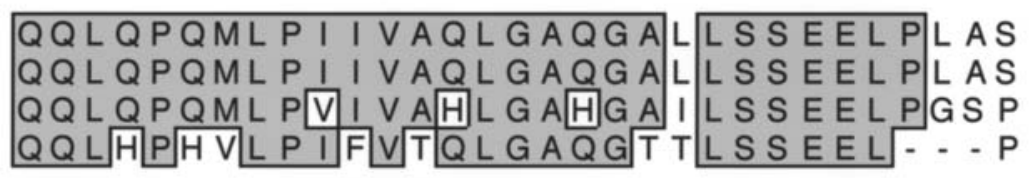 \\
\hline $\begin{array}{l}\text { ouse } \\
\text { uman }\end{array}$ & $\begin{array}{l}125 \\
121\end{array}$ & 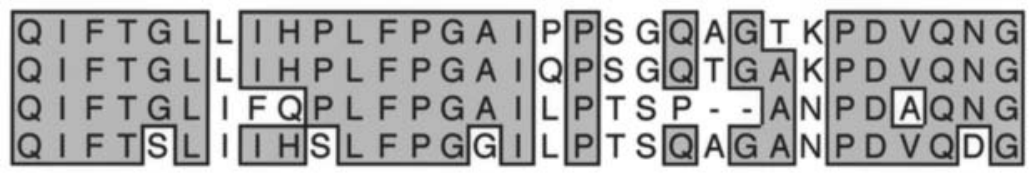 \\
\hline $\begin{array}{l}\text { Mouse } \\
\text { Rat } \\
\text { Dog } \\
\text { Human }\end{array}$ & $\begin{array}{l}155 \\
155 \\
154 \\
152\end{array}$ & 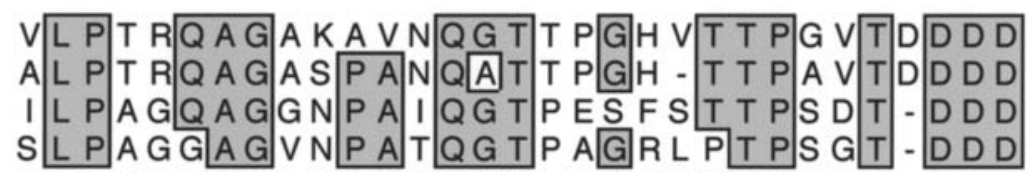 \\
\hline $\begin{array}{l}\text { og } \\
\text { uman }\end{array}$ & $\begin{array}{l}186 \\
185\end{array}$ & 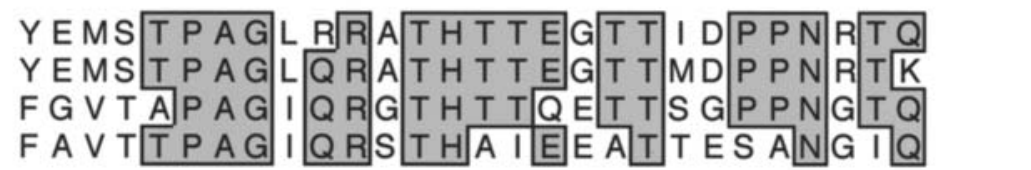 \\
\hline
\end{tabular}

A

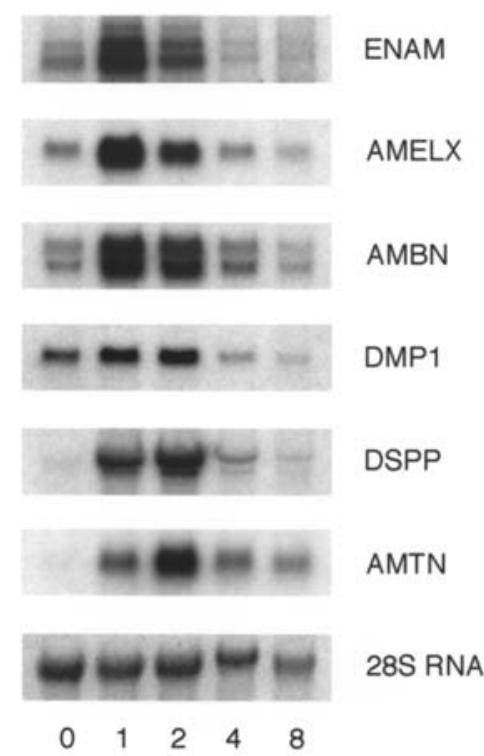

$\mathrm{B}$

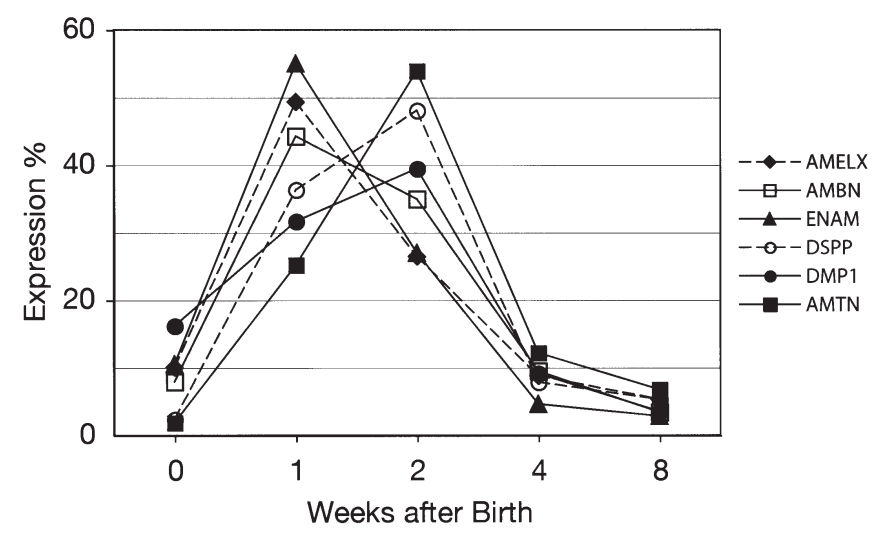

Figure 5. Expression of the secreted tooth-specific proteins in rat mandibles. Total RNA was extracted from rat mandibles of newborn rats $(0,1,2,4,8$ weeks after birth) and processed for Northern blotting. (A) The blots were hybridized sequentially with labeled cDNA clones for different proteins as indicated. (B) The signals were quantified with a phosphorimager and plotted for each transcript (total $=100 \%$ ). 


\section{Discussion}

The organic material of our teeth consists of a variety of extracellular matrix proteins that in concert provide the microenvironment for the controlled deposition of mineral crystals. Several research groups have used the elegant techniques of molecular biology to identify tooth-specific proteins. Matsuki et al (13) prepared a cDNA library from rat incisors and randomly sequenced 400 clones. Their study led to the discovery of ameloblastin as a novel component of enamel (14). Buchaille et al (15) used subtractive hybridization to generate a cDNA library from human odontoblast cultures. The authors sequenced 154 cDNA clones and found many transcripts for DSPP and ameloblastin. Dey et al (16) used suppression subtractive hybridization to identify gene products from odontoblasts that were not expressed in osteoblasts. The majority of the specific clones were found to code for DSPP. Hao et al (17) utilized a similar technique to identify genes that were expressed by immortalized odontoblasts, but not by osteoblasts. These authors found several novel genes, five of which were partially characterized in their report.

It is interesting to note that all of the studies mentioned above identified, in addition to the tooth-specific clones, a variety of gene products that are ubiquitously expressed in hard connective tissues (collagen I, osteonectin, osteocalcin) as well as several gene products that are barely expressed in teeth or bone (neuronal proteins, cerebellum proteins and odorant-binding proteins). These observations are in keeping with our findings that nearly $50 \%$ of the cDNA clones were barely expressed in teeth or bone. The relevance of these transcripts, if any at all, remains to be determined. The majority of the other clones were expressed at similar levels in teeth and bone, illustrating the close relationship of dentin and bone. Only a small percentage of the clones from our library were expressed specifically in teeth. Notably, in this group we also found typical bone proteins, such as collagen I, osteocalcin and alkaline phosphatase. Northern blotting experiments confirmed that these genes were transcribed at much higher levels in teeth than in bone. The high transcription rate might explain why clones for general bone proteins occurred in the tooth-specific library. In this context, one should remember that rat incisors grow at a rate of nearly $3 \mathrm{~mm}$ per week (8).

A transcript that was more abundant in teeth than in bone was the rat homologue of mouse FATSO. FATSO represents one of six genes that are deleted in the 'fused toes mouse', a mouse model that shows craniofacial abnormalities and syndactyly of the forelimbs $(18,19)$. The FATSO protein might play a role in the nucleus as its sequence harbors a nuclear localization signal. The fact that FATSO occurred in our library suggests that it may be involved in the development of teeth. However, FATSO transcripts have also been found in other tissues, including testis, kidney and muscle (18).

A protein expressed in teeth but not in bone was the intermediate filament protein cytokeratin 13. This is explained by the fact that cytokeratin 13 is usually expressed in stratified epithelia that line the oral cavity. The oral epithelium is the tissue from which ameloblasts are derived during tooth bud formation. Amelogenin can interact with cytokeratins, suggesting that cytokeratins may play a role in the organization of the extracellular matrix during amelogenesis (20).

The vast majority of our tooth-specific clones were found to belong to the family of the secreted, Ca-binding phosphoproteins. Seven out of the 8 described phosphoproteins from teeth and bone were identified in our cDNA library. Four (enamelin, amelogenin, ameloblastin, DSPP) were specifically expressed in teeth. Three (DMP1, osteopontin, bone sialoprotein) were expressed in both tissues. No clones for MEPE were found in our library, suggesting that MEPE is barely expressed in teeth. This is in line with the original observation that MEPE is synthesized primarily by bone marrow and by some tumor cells (21).

A novel gene product specifically expressed in teeth was identified as the rat homologue of mouse amelotin. This protein was recently discovered by Iwasaki et al (22) during the differential display of ameloblasts, odontoblasts and osteoblasts. Amelotin is specifically secreted by ameloblasts and appears to play a critical role during the maturation stage of the cells. Iwasaki et al (22) cloned and characterized amelotin from humans and mice. We identified the rat homologue, which encompasses 212 amino acid residues and which shows $62 \%$ sequence identity with the human homologue. To note, the gene for amelotin is situated on the long arm of human chromosome 4, adjacent to the gene for ameloblastin. It is therefore possible that amelotin also belongs to the family of the secreted phosphoproteins.

During the development of the mandible, the toothspecific genes showed distinct temporal expression patterns. Amelogenin, ameloblastin and enamelin were expressed at maximal levels one week after birth. The concomitant expression of these genes is explained by the fact that they are transcribed primarily by one cell type, the ameloblasts. The nearly identical shape of the expression curves might also suggest that the promoters of the three enamel genes are regulated in concert, although the enamelin gene is transcribed at a much lower level than the other two genes. In contrast, DSPP and DMP1 showed maximal expression two weeks after birth. These two gene transcripts are produced primarily by odontoblasts and reveal a unique colocalization in dentinal tubules and predentin as demonstrated with monoclonal antibodies (23). Amelotin, although transcribed exclusively by ameloblasts, exhibited maximal expression two weeks after birth similar to DSPP and DMP1. Since it was expressed considerably later than the other enamel genes it hardly plays a structural role comparable to those of ameloblastin, amelogenin and enamelin. It has therefore been suggested that amelotin might serve as a proteinase similar to kallikrein (22). However, no proteolytic activity has so far been demonstrated for recombinant amelotin from humans or mice.

\section{Acknowledgments}

This study was supported by grants from the Société Suisse d'Odonto-stomatologie SSO, the Foundation for Clinical Research and the ITI Foundation (382/2005).

\section{References}

1. Robey PG: Vertebrate mineralized matrix proteins: structure and function. Connect Tissue Res 35: 131-136, 1996. 
2. Butler WT: Dentin matrix proteins. Eur J Oral Sci 106 (suppl 1): 204-210, 1998.

3. Hu JC, Sun X, Zhang C and Simmer JP: A comparison of enamelin and amelogenin expression in developing mouse molars. Eur J Oral Sci 109: 125-132, 2001.

4. Fisher LW, Torchia DA, Fohr B, Young MF and Fedarko NS: Flexible structures of SIBLING proteins, bone sialoprotein, and osteopontin. Biochem Biophys Res Commun 280: 460-465, 2001.

5. Fisher LW and Fedarko NS: Six genes expressed in bones and teeth encode the current members of the SIBLING family of proteins. Connect Tissue Res 44 (suppl. 1): 33-40, 2003.

6. Kawasaki K and Weiss KM: Mineralized tissue and vertebrate evolution: the secretory calcium-binding phosphoprotein gene cluster. Proc Natl Acad Sci USA 100: 4060-4065, 2003.

7. Huq NL, Cross KJ, Ung M and Reynolds EC: A review of protein structure and gene organisation for proteins associated with mineralised tissue and calcium phosphate stabilisation encoded on human chromosome 4. Arch Oral Biol 50: 599-609, 2005.

8. Addison WHF and Appleton JL: The structure and growth of the incisor teeth of the albino rat. J Morphol 26: 42-96, 1915.

9. Chomczynski P and Sacchi N: Single-step method of RNA isolation by acid guanidinium thiocyanate-phenol-chloroform extraction. Anal Biochem 162: 156-159, 1987.

10. Ausubel FM, Brent R, Kingston RE, Moore DD, Seidman JG, Smith JA and Struhl K (eds). Current Protocols in Molecular Biology. Greene Publishing Associates, New York, 1987.

11. Feinberg AP and Vogelstein B: A technique for radiolabeling DNA restriction endonuclease fragments to high specific activity. Anal Biochem 132: 6-13, 1983.

12. Schild C and Trueb B: Mechanical stress is required for highlevel expression of connective tissue growth factor. Exp Cell Res 274: 83-91, 2002.

13. Matsuki Y, Nakashima M, Amizuka N, Warshawsky H, Goltzman D, Yamada KM and Yamada Y: A compilation of partial sequences of randomly selected cDNA clones from the rat incisor. J Dent Res 74: 307-312, 1994.
14. Krebsbach PH, Lee SK, Matsuki Y, Kozak CA, Yamada KM and Yamada Y: Full-length sequence, localization, and chromosomal mapping of ameloblastin. A novel tooth-specific gene. J Biol Chem 271: 4431-4435, 1996.

15. Buchaille R, Couble ML, Magloire H and Bleicher F: A substractive PCR-based cDNA library from human odontoblast cells: identification of novel genes expressed in tooth forming cells. Matrix Biol 19: 421-430, 2000.

16. Dey R, Son HH and Cho MI: Isolation and partial sequencing of potentially odontoblast-specific/enriched rat cDNA clones obtained by suppression subtractive hybridization. Arch Oral Biol 46: 249-260, 2001

17. Hao J, He G, Narayanan K, Zou B, Lin L, Muni T, Ramachandran A and George A: Identification of differentially expressed cDNA transcripts from a rat odontoblast cell line. Bone 37: 578-588, 2005.

18. Peters T, Ausmeier K and Ruther U: Cloning of Fatso (Fto), a novel gene deleted by the fused toes $(\mathrm{Ft})$ mouse mutation. Mamm Genome 10: 983-986, 1999.

19. Peters T, Ausmeier K, Dildrop R and Ruther U: The mouse fused toes $(\mathrm{Ft})$ mutation is the result of a $1.6-\mathrm{Mb}$ deletion including the entire Iroquois B gene cluster. Mamm Genome 13: 186-188, 2002.

20. Ravindranath RM, Tam WY, Nguyen P and Fincham AG: The enamel protein amelogenin binds to the $\mathrm{N}$-acetyl-Dglucosamine-mimicking peptide motif of cytokeratins. J Biol Chem 275: 39654-39661, 2000.

21. Rowe PS, de Zoysa PA, Dong R, Wang HR, White KE, Econs MJ and Oudet CL: MEPE, a new gene expressed in bone marrow and tumors causing osteomalacia. Genomics 67: 54-68, 2000.

22. Iwasaki K, Bajenova E, Somogyi-Ganss E, Miller M, Nguyen V, Nourkeyhani H, Gao Y, Wendel M and Ganss B: Amelotin - a novel secreted, ameloblast-specific protein. J Dent Res 84: $1127-1132,2005$

23. Baba O, Qin C, Brunn JC, Wygant JN, McIntyre BW and Butler WT: Colocalization of dentin matrix protein 1 and dentin sialoprotein at late stages of rat molar development. Matrix Biol 23: 371-379, 2004. 